\title{
ON THE PROPORTIONALITY OF CHERN AND RIEMANNIAN SCALAR CURVATURES
}

\author{
MICHAEL G. DABKOWSKI AND MICHAEL T. LOCK
}

\begin{abstract}
On a Kähler manifold there is a clear connection between the complex geometry and underlying Riemannian geometry. In some ways, this can be used to characterize the Kähler condition. While such a link is not so obvious in the nonKähler setting, one can seek to understand extensions of these characterizations to general Hermitian manifolds. This idea has been the subject of much study from the cohomological side, however, the focus here is to address such a question from the perspective of curvature relationships. In particular, on compact manifolds the Kähler condition is characterized by the relationship that the Chern scalar curvature is equal to half the Riemannian scalar curvature. What we study here is the existence, or lack thereof, of non-Kähler Hermitian metrics for which a more general proportionality relationship between these scalar curvatures holds.
\end{abstract}

\section{Contents}

1. Introduction

2. Background

3. The sKlsc equation

4. Schrödinger operators with scaled and warped potentials 14

5. Negative Gauduchon degree case 17

6. Positive Gauduchon degree case $\quad 22$

References

\section{InTRODUCTION}

In studying the geometry of complex manifolds, one often seeks Hermitian metrics with certain "desirable" properties. For instance, Kähler geometry provides a very deep and beautiful picture. While this has long been an exciting and fruitful area of research, the Kähler condition is somewhat restrictive. In the non-Kähler setting, unfortunately, a great deal of geometric structure and intuition is lost, and it is not yet completely clear what are the fundamental questions or geometric characteristics to investigate. Much of the existing work in this direction focuses either on metrics which satisfy certain structural conditions that are a weakening of those found in Kähler geometry, or on Hermitian analogues of classical Riemannian questions concerning

Date: June 21, 2016.

The second author was partially supported by NSF Grant DMS-1148490. 
curvature. For example, see [2, 11, 19, 6, 1]. The work of this paper can be viewed as somewhere between these two ideas as we study the weakening of a relationship between scalar curvatures that characterizes the Kähler condition.

In particular, we will be concerned with a relationship between the scalar curvatures resulting from what are, perhaps, the two most natural connections to consider in this setting - the Chern connection on the holomorphic tangent bundle, and the Levi-Civita connection on the underlying Riemannian manifold. The Riemannian and Chern scalar curvatures of a Hermitian manifold $(M, J, g)$ will be denoted by $S(g)$ and $S_{C}(g)$ respectively. These are the scalar curvatures corresponding to the LeviCivita and Chern connections. Many fundamental questions in Riemannian geometry concern the Riemannian scalar curvature, which provides one of the most simple and intuitive geometric pictures of the underlying manifold as it arises in the asymptotic expansion of the volume of geodesic balls, see [3]. The motivation for studying Chern scalar curvature here, as opposed to a variety of other complex curvature scalars, comes mainly from the naturalness of the connection and the resulting complex geometry, as well as it's interplay with the underlying real geometry. It is worthwhile to note, though, that there has been much other recent interest in problems regarding Chern curvatures; for instance, in non-Kähler Calabi-Yau problems and the Chern Yamabe problem, see [21, 1].

On a compact Hermitian manifold, the Chern and Riemannian scalar curvatures satisfy the relationship $S(g)=2 \cdot S_{C}(g)$ if and only if the metric is Kähler [18, 12]. In fact, if $S(g)$ and $2 \cdot S_{C}(g)$ are even equal in average over the manifold, then the metric must be Kähler. Therefore, on a compact manifold, this relationship of scalar curvatures can be seen to characterize the Kähler condition. One natural question to ask is whether, on a non-compact manifold, there can exist non-Kähler Hermitian metrics for which the relationship of scalar curvatures $S(g)=2 \cdot S_{C}(g)$ holds. In [4], the authors examined this question for $\mathrm{U}(n)$-invariant Hermitian metrics on annuli in $\mathbb{C}^{n}$, and showed that every conformal class admits a 1-dimensional family (up to scale) of non-Kähler Hermitian metrics with the property that $S(g)=2 \cdot S_{C}(g)$. Furthermore, the authors classified the space of all such metrics on these annuli.

What we will study here is the existence of non-Kähler Hermitian metrics on compact complex manifolds, which are always assumed to be without boundary, for which a generalization of the scalar curvature relationship that characterizes the Kähler condition in the compact setting holds. Specifically, we ask whether there can exist Hermitian metrics on compact complex manifolds which posses the quality that $S(g)=2 \kappa \cdot S_{C}(g)$ for some constant $\kappa$. Such metrics would necessarily be non-Kähler for $\kappa \neq 1$. It is interesting to note that if such metrics exist, then a simple and intuitive picture of the underlying geometry can be extracted from the Chern scalar curvature. More specifically, we make the following definition.

Definition 1.1. A Hermitian metric $g$ on a complex manifold $(M, J)$ is said to be a scaled Kähler like scalar curvature (sKlsc) metric if

$$
S(g)=2 \kappa \cdot S_{C}(g)
$$

for some constant $\kappa \neq 0$. The associated constant $\kappa$ is called the scaling constant. 
Remark 1.2. We require that $\kappa \neq 0$ because, if $\kappa=0$, no intuition for the Chern scalar curvature would be derived from this relationship as the question is just that of existence for a Riemannian-scalar-flat metric.

For certain reasons, which will become apparent, it is interesting to study the existence of sKlsc metrics within a conformal class. On a Hermitian manifold $(M, J, g)$, it is assumed that the complex structure is fixed and that any metric conformal to $g$ is Hermitian with respect to it.

The sKlsc Problem: In a Hermitian conformal structure on a compact complex manifold, of complex dimension $n \geq 2$, does there exist an sKlsc representative?

We are able to address this question in the case where the conformal class contains a balanced representative, which we denote by $g_{b}$. This is because, when there is not a balanced representative, the operator we study becomes non-self-adjoint. The sKlsc problem differs significantly depending upon whether or not the balanced representative is actually a Kähler metric. What we will see is that, when there is a Kähler representative, there are fewer existence results but clear uniqueness results, and, when there is not a Kähler representative, there are more broad existence results but less clear uniqueness results. The nature of the sKlsc question also very much depends upon the Gauduchon degree of the conformal class. In particular, the negative Gauduchon degree case depends upon the behavior of a relationship of scalar curvatures around an analytically degenerate instantiation of the problem. Specifically, it splits into two cases, depending upon whether or not the the scalar curvatures at the balanced representative, $g_{b}$, satisfy

$$
S\left(g_{b}\right)-2\left(\frac{2 n-1}{n}\right) S_{C}\left(g_{b}\right) \geq 0
$$

with

$$
\left.S\left(g_{b}\right)\right|_{p}-\left.2\left(\frac{2 n-1}{n}\right) S_{C}\left(g_{b}\right)\right|_{p}=0 \text { at least at one point } p \in M
$$

where

$$
\left.S\left(g_{b}\right)\right|_{p} \neq 0 \text { and }\left.S_{C}\left(g_{b}\right)\right|_{p} \neq 0 .
$$

It is only possible to establish non-trivial existence results in for Hermitian conformal structures with non-zero Gauduchon degree, see Theorem 1.4 below. For the purpose of clarity of exposition, we give a broad picture of existence for sKlsc metrics for these cases in Theorem 1.3. Subsequently, in Section 1.1 and Section1.2, a detailed account of these results is provided.

Theorem 1.3. Let $[g]$ be a Hermitian conformal structure on a compact complex manifold of complex dimension $n \geq 2$, which contains a balanced representative $g_{b}$. If the Gauduchon degree is

- positive, then there exists at least one sKlsc representative of $[g]$ and every such metric has scaling constant $\kappa \in(-\infty, 1]$. 
- negative, and condition (1.1)

(1) is not satisfied, then there exists at least one sKlsc representative of $[g]$ and every such metric has scaling constant $\kappa \in[1, \infty)$.

(2) is satisfied, then the only scaling constant for which there can possibly exist a non-Kähler sKlsc representative of $[g]$ is $\kappa=\frac{2 n-1}{n}$. Furthermore, unless $S\left(g_{b}\right)-2\left(\frac{2 n-1}{n}\right) S_{C}\left(g_{b}\right)$ vanishes at least twice on the manifold and $S_{C}\left(g_{b}\right) \leq 0$, there can be no such metric.

The proof of this result, and the corresponding more detailed results given in Section 1.1 and Section 1.2, begins with the construction and examination of a 1parameter family of second order semi-linear PDEs in the scaling constant $\kappa$. Outside of $\kappa=\frac{2 n-1}{n}$, the problem transforms into a family of eigenvalue problems for Schrödinger operators on compact manifolds. The subsequent study then separates into several distinct regimes depending upon how the geometry of the balanced manifold behaves in the neighborhood of two critical scaling constants, $\kappa=1$ and $\kappa=\frac{2 n-1}{n}$. We employ our previous results from [5] to study the eigenvalue behavior in the majority of these cases. However, in one particular situation it is necessary to deal with a family of Schrödinger operators that has both a scaling and a warping of the potential function. We examine this question separately, and in a general setting, in Section 4, and prove a surprising lack of stability in behavior. There is however, one analytically degenerate case. This is for negative Gauduchon degree and the scaling constant $\kappa=\frac{2 n-1}{n}$. For this scaling constant, the second order PDE degenerates into a first order non-linear autonomous Hamilton-Jacobi equation. The existence of a classical solution to such an equation on a compact manifold is very rare. It is common to instead seek a viscosity solution which in general has lower regularity. Not only is this lower regularity not suitable for our purposes here, but, interesting, for the particular equation arising in this problem, the existence of a viscosity solution would be incredibly rare as well.

Theorem 1.3 is stated to include the possibility of Kähler metrics, which are clearly sKlsc, existing in the conformal class. While this gives the most general picture of existence for these metrics, the true question to be dealt with is that for non-Kähler sKlsc metrics. The specific results are detailed in Section 1.1 for conformal classes which contain a Kähler representative, and in Section 1.2 for conformal classes which contain a balanced metric but no Kähler representative.

Interestingly, while sKlsc metrics are guaranteed to exist in almost every conformal class of non-zero Gauduchon degree, the sKlsc condition in a conformal class of zero Gauduchon degree is equivalent to the Kähler condition. Specifically, we have the following result.

Theorem 1.4. Let $[g]$ be a Hermitian conformal structure on a compact complex manifold, of complex dimension $n \geq 2$, of zero Gauduchon degree. There does not exist an sKlsc representative unless the conformal class contains a Kähler representative in which case that is the only sKlsc representative. 
While Theorem 1.4 addresses the sKlsc question completely for the case of zero Gauduchon degree, the techniques we have developed to study it in the case of nonzero Gauduchon degree require the existence of a balanced metric in the conformal class to eliminate the non-self-adjoint issue. It would be interesting to study this question in full generality.

1.1. When $[g]$ contains a Kähler representative. Here we seek to understand the existence of non-Kähler sKlsc metrics in conformal classes which contain a Kähler representative, $g_{k}$. Then, if such metrics do exist, what are the associated scaling constants, and for each such constant is the metric unique up to scale? The answers depend significantly upon the behavior of the scalar curvature of the Kähler representative. Since $S\left(g_{k}\right)=2 \cdot S_{C}\left(g_{k}\right)$, the scalar curvature conditions will be stated simply in terms of $S_{C}\left(g_{k}\right)$. Recall, by virtue of Theorem [1.4, it is only necessary to study the non-zero Gauduchon degree cases. The sKlsc problem is addressed for negative and positive Gauduchon degrees in Theorem 1.5 and Theorem 1.7 respectively.

Theorem 1.5. Let $[g]$ be a Hermitian conformal structure of negative Gauduchon degree on a compact complex manifold of complex dimension $n \geq 2$. If $[\mathrm{g}]$ contains a Kähler representative, $g_{k}$, and

(1) $S_{C}\left(g_{k}\right)$ changes sign, then there are exactly two non-Kähler sKlsc representatives of $[g]$ up to scale. These have scaling constants, $\kappa_{1}$ and $\kappa_{2}$, which lie in the respective regimes

$$
1<\kappa_{1}<\frac{2 n-1}{n} \text { and } \frac{2 n-1}{n}<\kappa_{2}<\infty,
$$

and satisfy the relationship

$$
\left(1-\kappa_{1}\right)\left(1-\kappa_{2}\right)=\frac{(n-1)^{2}}{n^{2}}
$$

Furthermore, letting $e^{-2 f_{\kappa_{1}}}$ and $e^{-2 f_{\kappa_{2}}}$ denote the conformal factors by which the respective sKlsc metrics are obtained, normalized to unit length in $L^{2}$, then these conformal factors satisfy the relationship

$$
e^{-2 f_{\kappa_{2}}}=e^{-2(1-n) f_{\kappa_{1}}} \text {. }
$$

(2) $S_{C}\left(g_{k}\right) \leq 0$, then the only scaling constant for which there can possibly exist a non-Kähler sKlsc representative of $[g]$ is $\kappa=\frac{2 n-1}{n}$. Furthermore, unless $S_{C}\left(g_{k}\right)$ vanishes at least twice on the manifold, no such representatives exist.

Remark 1.6. Regarding the existence result of Theorem 1.5 part (11), it is important to note that while the possible range of the scaling constants $\kappa_{1}$ and $\kappa_{2}$ are the same for any compact Kähler manifolds satisfying these scalar curvature conditions, the actual values of these scaling constants almost certainly differs between such manifolds. In fact, for each particular such manifold, the possible ranges for $\kappa_{1}$ and $\kappa_{2}$ can actually be bounded further away from 1 and $\infty$ respectively. However, we do not state these better bounds explicitly as their values have quite a complicated expression in terms of the total scalar curvature, the $L^{\infty}(M)$ norm of the scalar curvature, volume of 
the manifold and the Poincaré constant of the manifold. For the interested reader, they can be obtained by substituting $\frac{(1-\kappa)(2 n-1)(n-1)}{(n \kappa+1-2 n)^{2}} S_{C}\left(g_{k}\right)$ for $\mathcal{V}$ in the inequality of Corollary 2.1 and solving for $\kappa$.

Theorem 1.7. Let $[g]$ be a Hermitian conformal structure of positive Gauduchon degree on a compact complex manifold of complex dimension $n \geq 2$. If $[\mathrm{g}]$ contains a Kähler representative, $g_{k}$, and

(1) $S_{C}\left(g_{k}\right)$ changes sign, then the only scaling constants for which a non-Kähler sKlsc representative of $[g]$ can possibly exist are in the regime $(-\infty, 1)$. Furthermore, if

$$
\frac{2 n-1}{4 n} \leq \frac{\int_{M} S_{C}\left(g_{k}\right)}{P\left\|S_{C}\left(g_{k}\right)\right\|_{\infty}\left(4 \operatorname{Vol}(M)\left\|S_{C}\left(g_{k}\right)\right\|_{\infty}+\int_{M} S_{C}\left(g_{k}\right)\right)},
$$

where $P$ is the Poincaré constant, then the sKlsc problem has no solution.

(2) $S_{C}\left(g_{k}\right) \geq 0$, then no non-Kähler sKlsc representatives of $[g]$ exist.

The same techniques used to prove Theorem 1.5 part (11) are not entirely applicable in this situation as there is a certain bound on the potential function of the Schrödinger operator formulation of the equation $S(g)=2 \kappa \cdot S_{C}(g)$. However, this does not preclude the existence of a conformal non-Kähler sKlsc metric for some $\kappa<1$. In fact, there are certain examples for which we know such a metric must exist (See Section 6.2 for more details). We suspect, though, that there are some compact Kähler manifolds, that satisfy these scalar curvature conditions, for which no conformal non-Kähler sKlsc metric exists. It would be interesting to investigate what further properties of the initial Kähler manifold this may depend upon.

1.2. When $[g]$ does not contain a Kähler representative. Here we seek to understand the existence, or lack thereof, for sKlsc metrics in conformal classes which do not contain a Kähler representative. The question here differs significantly from the situation where there does exist a Kähler representative, addressed above in Section 1.1, in two primary ways - the existence of a non-self-adjoint first order term, and a warping of the potential function in the Schrödinger operator formulation of the sKlsc equation. The latter of these two issues impacts the case of negative Gauduchon degree as it leads to a surprising lack of stability for eigenvalues in an associated family of Schrödinger operators. We study this phenomenon, in a general sense, in Section 4. Since any sKlsc metric in a conformal class of zero Gauduchon degree must be Kähler, recall Theorem 1.4, we only need ask the sKlsc question here for negative and positive Gauduchon degrees. These are addressed in Theorem 1.8 and Theorem 1.10 respectively. It is interesting to contrast the results here with those corresponding in Section 1.1, and comments are made accordingly.

Theorem 1.8. Let $[g]$ be a Hermitian conformal structure of negative Gauduchon degree on a compact complex manifold of dimension $n \geq 2$. If $[g]$ contains a balanced representative, $g_{b}$, but not a Kähler representative, and 
(1) $\int_{M} S\left(g_{b}\right)-2\left(\frac{2 n-1}{n}\right) S_{C}\left(g_{b}\right)>0$, where $S\left(g_{b}\right)-2\left(\frac{2 n-1}{n}\right) S_{C}\left(g_{b}\right)$

(a) changes sign, then there exists at least one sKlsc representative which has scaling constant in the regime $\left(\frac{2 n-1}{n}, \infty\right)$.

(b) is non-negative, and the additional conditions of (1.1) are not satisfied, then there exists at least one sKlsc representative and every such metric has scaling constant in the regime $\left(1, \frac{2 n-1}{n}\right)$.

(c) is non-negative, and the additional conditions of (1.1) are satisfied, then the only scaling constant for which a non-Kähler sKlsc representative can possibly exist is $\kappa=\frac{2 n-1}{n}$. Furthermore, unless $S\left(g_{b}\right)-2\left(\frac{2 n-1}{n}\right) S_{C}\left(g_{b}\right)$ vanishes at least twice on the manifold, no such representative exists.

(2) $\int_{M} S\left(g_{b}\right)-2\left(\frac{2 n-1}{n}\right) S_{C}\left(g_{b}\right)=0$, where $S\left(g_{b}\right)-2\left(\frac{2 n-1}{n}\right) S_{C}\left(g_{b}\right)$

(a) changes sign, then there exists at least one sKlsc representative and every such metric has scaling constant in the regime $\left(\frac{2 n-1}{n}, \infty\right)$.

(b) does not change sign, then the balanced representative is the unique sKlsc representative of $[\mathrm{g}]$ and it has scaling constant $\kappa=\frac{2 n-1}{n}$.

(3) $\int_{M} S\left(g_{b}\right)-2\left(\frac{2 n-1}{n}\right) S_{C}\left(g_{b}\right)<0$, then there exists at least one sKlsc representative and every such metric has scaling constant in the regime $\left(1, \frac{2 n-1}{n}\right)$.

Remark 1.9. In Theorem 1.8 (1a), while at least one sKlsc metric with scaling constant in the regime of $\left(\frac{2 n-1}{n}, \infty\right)$ is guaranteed to exist, in certain situations there may also exist sKlsc metrics with scaling constants in the regime $\left(1, \frac{2 n-1}{n}\right)$. Also, recall the discussion following Theorem 1.3 regarding Theorem 1.8 (1c).

Considering Theorem [1.8 in light of the analogous result for the case that the conformal class contains a Kähler representative, Theorem 1.5, we see here that, while there is a broader range of existence results, there specificity of the analogous situation is lacking. This is due to a wider possible range of behaviors of the warped potential function in the Schrödinger operator formulation of the sKlsc equation.

In the case of positive Gauduchon degree, the distinction between broadness of existence is even more extreme. While in Theorem 1.7 we see that existence of non-Kähler sKlsc metrics is very rare when the conformal class contains a Kähler representative, in Theorem 1.10 below we find that such a metric is always guaranteed to exist in this situation.

Theorem 1.10. Let $[g]$ be a Hermitian conformal structure of positive Gauduchon degree on a compact complex manifold of dimension $n \geq 2$. If $[g]$ contains a balanced representative, $g_{b}$, but not a Kähler representative, then there exists at least one sKlsc representative and every such metric has scaling constant in the regime $(-\infty, 1)$. 
1.3. Acknowledgements. The authors would like to thank Joseph Conlon and Lorenzo Sadun for many useful remarks, as well as John Gardner and Oliver Kelavan for many interesting conversations regarding the physical implications of this work. Also, we would like to thank Jessica Dabkowski for putting up with us, which will be good practice for dealing with children.

\section{BACKGROUND}

This section is composed to two parts which contain summaries of the necessary background in complex geometry and Schrödinger operators respectively. Further references are given in each section.

2.1. Hermitian manifolds and scalar curvatures. A brief description of the background necessary for sKlsc component of this work is provided in this section. In particular, Hermitian manifolds, the Chern connection, its curvature, and the transformations of the Riemannian and Chern scalar curvatures under a conformal change are discussed. For a more detailed account of these topics see [15, 3, 17, 1].

A Riemannian metric $g$, on a complex manifold $(M, J)$, is said to be Hermitian if $g(J X, J Y)=g(X, Y)$ for any vector fields $X$ and $Y$. The associated 2-form of a Hermitian metric, $\omega \in \Lambda^{1,1}$, is defined by $\omega(X, Y)=g(J X, Y)$, and can be written as

$$
\omega=\sqrt{-1} \sum_{i, j} g_{i \bar{j}} d z_{i} \wedge d \bar{z}_{j}, \quad \text { where } g_{i \bar{j}}=g\left(\frac{\partial}{\partial z_{i}}, \frac{\partial}{\partial \bar{z}_{j}}\right) \text {. }
$$

A Hermitian metric is called Kähler if its associated 2-form is closed.

On a Hermitian manifold $(M, J, g)$, there exists a unique connection on the holomorphic tangent bundle $T^{1,0} M$, known as the Chern connection, which is compatible with the Hermitian metric and the complex structure. The bundles $T^{1,0} M$ and $T M$ are naturally isomorphic, which allows for a comparison between the Chern connection and the Levi-Civita connection. While both are compatible with the metric, the Chern connection does not necessarily induce a symmetric connection on $T M$ and the Levi-Civita connection does not necessarily induce a connection compatible with the complex structure. If these connections do satisfy the additional condition of the other, they are equal since both are unique. This occurs if and only if $g$ is Kähler.

While knowledge of Riemannian geometry is assumed here, many readers may not be familiar with the Chern curvatures tensors (i.e. those with respect to the Chern connection) in the non-Kähler setting. Thus, a brief description is provided, see [15, 18, for more detail. Let $(M, J, g)$ be a Hermitian manifold, of complex dimension $n$, with associated 2-form $\omega$. The components of the full Chern curvature tensor on the Hermitian holomorphic vector bundle $\left(T^{1,0} M, g\right)$ are

$$
\Theta(g)_{i \bar{j} k \bar{l}}=-\frac{\partial^{2} g_{k \bar{l}}}{\partial z_{i} \partial \bar{z}_{j}}+g^{p \bar{q}} \frac{\partial g_{p \bar{l}}}{\partial \bar{z}_{j}} \frac{\partial g_{k \bar{q}}}{\partial z_{i}} .
$$

The Chern-Ricci curvature,

$$
\Theta(g)_{i \bar{j}}=g^{k \bar{l}} \Theta(g)_{i \bar{j} k \bar{l}}
$$


is obtained by tracing on the $k$ and $\bar{l}$ indices, and the Chern scalar curvature,

$$
S_{C}(g)=g^{i \bar{j}} \Theta(g)_{i \bar{j}}
$$

by tracing further on the $i$ and $\bar{j}$ indices. The Chern-Ricci curvature has the associated Chern-Ricci form given by $\rho=\sqrt{-1} \sum_{i, j} \Theta(g)_{i \bar{j}} d z_{i} \wedge d \bar{z}_{j}=-\sqrt{-1} \partial \bar{\partial} \log \operatorname{det}\left(g_{i \bar{j}}\right)$.

The focus of this paper is to study the existence of Hermitian metrics on compact manifolds for which a certain desired relationship between the Chern and Riemannian scalar curvatures hold. It was shown in [18] that these are related by

$$
S(g)=2 S_{C}(g)+\left\langle\partial \partial^{*} \omega, \omega\right\rangle+\left\langle\overline{\partial \partial}^{*} \omega, \omega\right\rangle-2\left|\partial^{*} \omega\right|^{2}-\frac{1}{2}|T(g)|^{2},
$$

where $T(g)$ is the torsion tensor. A relationship between these scalar curvatures was also found in terms of Lee forms in [12]. Extensive use will be made of the transformations of the Riemannian and Chern scalar curvatures under a conformal change of the metric which are recalled as follows. Consider the conformal change $\tilde{g}=e^{2 f} g$. It is well-known, for a real $2 n$-dimensional manifold, that the Riemannian scalar curvature of this conformal metric is given by

$$
S(\tilde{g})=e^{2 f}\left(2(2 n-1) \Delta f-2(2 n-1)(n-1)|\nabla f|^{2}+S(g)\right),
$$

where $\Delta$ denotes the Laplace-Beltrami operator with sign convention so that the spectrum is non-positive [3]. The Chern scalar curvature of this conformal metric is given by

$$
S_{C}(\tilde{g})=e^{2 f}\left(n \Delta f-n\langle d f, \theta\rangle_{\omega}+S_{C}(g)\right),
$$

where $\theta$ is the Lee form, or torsion 1-form, associated to $\omega$, which is defined by $d \omega^{n-1}=\theta \wedge \omega^{n-1}$. For a balanced Hermitian metric, i.e. a Hermitian metric for which the Lee form vanishes, the transformation of the Chern scalar curvature simplifies accordingly. In particular, note that Kähler metric are necessarily balanced. See [1] for an excellent reference regarding the conformal transformation of $S_{C}(g)$.

2.2. Schrödinger operators on compact manifolds. The Schrödinger equation arises in an astonishing number of both mathematical and physical problems, and while its origins lie in the study of quantum mechanics, see [20] and references therein, it has driven an incredible mathematical theory itself.

On a Riemannian manifold, the time independent Schrödinger equation is given by

$$
L_{\mathcal{V}}(\varphi)=-\Delta \varphi+\mathcal{V} \varphi=\lambda \varphi,
$$

where $\Delta$ is the Laplace-Beltrami operator with non-positive spectrum, $\mathcal{V}$ is a smooth potential and $\lambda$ is a constant. It is of both physical and mathematical interest to understand the negative eigenvalues (bound states) of the Schrödinger operator $L_{\mathcal{V}}$, in particular the lowest eigenvalue (ground state). On compact manifolds, the lowest eigenvalue is found by minimizing the Rayleigh quotient

$$
\min _{0 \neq \emptyset \in H^{1}} \frac{\int_{M}|\nabla \varphi|^{2}+\mathcal{V} \varphi^{2}}{\int_{M} \varphi^{2}} \text {. }
$$


Many of the classical results fail to hold in the compact setting due to a failure of certain heat kernel estimates. Recently, there has been some work on eigenvalue problems in this setting [14, 13, 8].

On a compact manifold, the only positive eigenfunction is that associated to the lowest eigenvalue, which is unique up to scale. For the work of this paper, it will be essential to understand when the lowest eigenvalue of such operators on compact manifolds is zero (when the ground state has zero energy). It is relatively simple to ascertain the sign of the lowest eigenvalue for every potential $\mathcal{V}$ except when

(i) $\mathcal{V}$ is smooth and changes sign on the manifold

$$
\text { (ii) } \int_{M} \mathcal{V}>0
$$

The question of sign for the lowest eigenvalue in this case is subtle, particularly that of if the lowest eigenvalue can be positive. The authors developed the following estimate which will be used frequently throughout this work.

Theorem 2.1 ([5], Theorem 1.1). Let $(M, g)$ be a compact manifold, and consider the Schrödinger operator $L_{\mathcal{V}}=-\Delta+\mathcal{V}$ with the potential $\mathcal{V}$ satisfying (2.10). If

$$
\frac{P\|\mathcal{V}\|_{\infty}\left(4 \operatorname{Vol}(M)\|\mathcal{V}\|_{\infty}+\int_{M} \mathcal{V}\right)}{\int_{M} \mathcal{V}} \leq 1
$$

where $P$ is the Poincaré constant, then the lowest eigenvalue of $L_{\mathcal{V}}$ is strictly positive.

It is important to the work of this paper to understand when the lowest eigenvalue is zero. Certainly Theorem 2.1 provides an obstruction to this, but even for such an operator $L_{\mathcal{V}}$ that does not satisfy the conditions of Theorem 2.1, it is unlikely that zero is contained in the spectrum, and even less likely that it is the lowest eigenvalue. In [5], the authors studied this lowest eigenvalue question under scalings of the manifold given a fixed potential. Since the Laplace-Beltrami operator scales inversely to the scaling of the metric, the question is equivalent to one under scalings of the potential. More specifically, the authors studied the following generalization of such a scaling. On a compact manifold, let $\mathcal{V}$ be a fixed potential satisfying (2.10), and consider the 1-parameter family positive scaling multipliers $f(t)$, where $f: \mathbb{R}^{+} \rightarrow \mathbb{R}^{+}$is positive continuous function satisfying

(i) $\lim _{t \rightarrow 0} f(t)=0$

(ii) $\lim _{t \rightarrow \infty} f(t)=\infty$.

In this setting, the authors proved the following theorem.

Theorem 2.2 ([5], Theorem 1.2). Let $(M, g)$ be a compact manifold and consider the 1-parameter family of Schrödinger operators

$$
L_{f(t) \mathcal{V}}=-\Delta+f(t) \mathcal{V}
$$

where $\mathcal{V}$ and $f(t)$ satisfy (2.10) and (2.11) respectively. There exists a $t^{*}>0$ for which

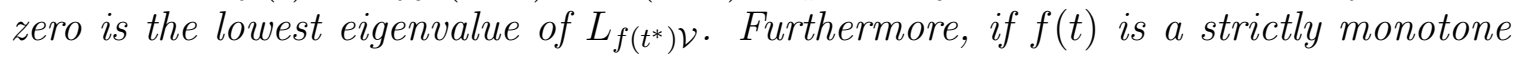
increasing function, then there is a unique such $t^{*}$. 
Remark 2.3. The lowest eigenvalue of $L_{f\left(t^{*}\right) \mathcal{V}}$ being zero is equivalent to the existence of a positive solution to $L_{f\left(t^{*}\right) \mathcal{V}}(\varphi)=0$, and that for each such $t^{*}$ this solution is unique up scale. A positive solution to $L_{f\left(t^{*}\right) \mathcal{V}}(\varphi)=0$, for $t^{*}>0$, is necessarily non-constant.

Remark 2.4. In fact, it was shown that there are regimes over which the lowest eigenvalue is positive and regimes when it is negative, see [5, Theorem 1.2] in its entirety. Under such a scaling Theorem 2.1 holds to show that there exists a positive regime. We see there is a negative regime by examining the Rayleigh quotient (2.9) using a test function with compact support in the region of the manifold where $\mathcal{V}<0$. Clearly, for $f(t)$ large enough this will be negative (See [5], Proposition 3.1).

The full generality of our work on the sKlsc problem here actually requires the analysis of a family of Schrödinger operators that have both a scaling and a warping of the potential function. Specifically, families of operators of the form

$$
-\Delta+f(t)\left(\mathcal{V}_{1}+h(t) \mathcal{V}_{2}\right)
$$

will be studied, where $\mathcal{V}_{1}, \mathcal{V}_{2} \in C^{\infty}(M)$ are fixed potentials, and $f(t)$ and $h(t)$ are scaling and warping functions respectively. Largely, the behavior of the eigenvalues of such families operators is stable and the above results, for when there is only a scaling of the potential, can be utilized in the analysis. However, in a certain circumstance, which is encountered in the studying of sKlsc question, there is actually a loss of stability for the operators in question. The lack of stability is not immediately obvious and requires additional analysis. This is done in Section 4.

\section{The sKlsc EquATION}

Here we understand the sKlsc relationship $S(g)-2 \kappa \cdot S_{C}(g)$ as a 1-parameter family of partial differential equations over the conformal class of the metric in the parameter of the scaling constant $\kappa$. Next, certain restrictions on the set of admissible scaling constants depending upon the Gauduchon degree of the conformal class are established. Subsequently, we make a change of variables, depending upon the parameter $\kappa$, which transforms the original family of equations into a 1-parameter family of Schrödinger type equations which have both a scaling and a warping of the potential. In most cases, the behavior of the lowest eigenvalues of this family of operators is stable. However, in one particular case, which is germane to the sKlsc problem, it is not clear whether or not the behavior is stable. We develop the necessary theory around this in Section 4, and show that there is, in fact, a lack of stability in certain circumstances.

Let $(M, J, g)$ be a compact Hermitian manifold of complex dimension $n \geq 2$. Consider the conformal metric $\tilde{g}=e^{-2 f} g$, and recall that we seek a conformal metric such that

$$
S(\tilde{g})-2 \kappa \cdot S_{C}(\tilde{g})=0 .
$$

We will refer to (3.1) as the sKlsc equation. In this section, the sKlsc equation will be formulated as a partial differential equation in the conformal factor $f$. Subsequently, the question of whether there are certain conditions that either restrict the possible 
range of scaling constants, $\kappa$, for which there is potentially a solution to (3.1), or provide an obstruction to the existence of any solution to the sKlsc problem altogether, is addressed. Recall that our interest is in non-Kähler sKlsc metrics, which are equivalent to sKlsc metrics with scaling constants $\kappa \neq 1$. Thus, for the remainder of this section, it is assumed that $\kappa \neq 1$.

Using the transformations of the Chern and Riemannian scalar curvatures under the conformal change of the metric $\tilde{g}=e^{-2 f} g$ for $f \in C^{\infty}(M)$, recall (2.6) and (2.7), the sKlsc equation can be formulated as the semi-linear PDE

$$
(2 n-1-n \kappa) \Delta f-(2 n-1)(n-1)|\nabla f|^{2}+n \kappa\langle d f, \theta\rangle_{\omega}+\frac{1}{2}\left(S(g)-2 \kappa \cdot S_{C}(g)\right)=0 .
$$

As $\kappa$ is allowed to vary, what we actually obtain is a 1-parameter family of secondorder semi-linear PDEs in the scaling constant. Notice that the corresponding elliptic operators are not self-adjoint, due to the $\langle d f, \theta\rangle_{\omega}$ term, unless the Lee form $\theta$ vanishes in which case the reference metric, $g$, is necessarily balanced.

Observe, as follows, that it is straightforward to obtain certain simple restrictions on when it is possible for solutions to the sKlsc problem to exist. As a reference metric, choose the unique (up to homotheties) Gauduchon metric in the conformal class, $g_{G}$. Since $M$ is compact, and the Lee form of the Gauduchon metric is co-closed, the non-self-adjoint first order term vanishes under integration and we see that any solution $f$ must satisfy

$$
\int_{M}|\nabla f|^{2}=\frac{1}{2(2 n-1)(n-1)} \int_{M} S\left(g_{G}\right)-2 \kappa \cdot S_{C}\left(g_{G}\right) .
$$

From (2.5), notice that $\int_{M} S\left(g_{G}\right)-2 S_{C}\left(g_{G}\right)<0$. Thus, if there is one or more solutions to the sKlsc problem, one finds that the range of possible scaling constants is restricted as follows:

$$
\text { If the Gauduchon degree of the conformal class is }\left\{\begin{array}{ll}
\text { negative } & \kappa>1 \\
\text { positive } & \kappa<1
\end{array}\right. \text {. }
$$

Remark 3.1. In the case that the Gauduchon degree of the conformal class is zero, there are no solutions to the sKlsc problem other than Kähler metrics. This is seen by rewriting the right hand side of (3.3) as

$$
\begin{aligned}
\int_{M} S\left(g_{G}\right)-2 \kappa \cdot S_{C}\left(g_{G}\right) & =(2-2 \kappa) \int_{M} S_{C}\left(g_{G}\right)+\int_{M} S\left(g_{G}\right)-2 S_{C}\left(g_{G}\right) \\
& =\int_{M} S\left(g_{G}\right)-2 S_{C}\left(g_{G}\right) \leq 0
\end{aligned}
$$

since $\int_{M} S_{C}\left(g_{G}\right)=0$ for the Gauduchon metric. Thus (3.3) can only hold if both sides vanish, in which case the initial metric must be Kähler. 
Notice, when $\kappa=\frac{2 n-1}{n}$, the coefficient of the Laplacian term in (3.2) vanishes and sKlsc equation degenerates into the first-order non-linear PDE

$$
|\nabla f|^{2}-\frac{1}{n-1}\langle d f, \theta\rangle_{\omega}=\frac{S(g)-2\left(\frac{2 n-1}{n}\right) S_{C}(g)}{2(2 n-1)(n-1)} .
$$

Therefore, the study of the sKlsc equation separates into the following two cases:

$$
\begin{aligned}
& \text { - When } \kappa \neq \frac{2 n-1}{n} \\
& \text { - When } \kappa=\frac{2 n-1}{n} \text {. }
\end{aligned}
$$

The scaling constant $\kappa=\frac{2 n-1}{n}$ will be referred to as the degenerate scaling constant. There are many obstructions to the existence of sKlsc metrics for this degenerate scaling constant. In particular, if the conformal class contains a balanced representative, then the sKlsc problem for $\kappa=\frac{2 n-1}{n}$ is obstructed unless $S_{C} \leq 0$ and not identically zero. This degenerate case will be discussed below in Section 5.3 . For the remainder of this section, it is assumed that $\kappa \neq \frac{2 n-1}{n}$, and the sKlsc equation (3.2) will be examined accordingly.

Because we are now assuming that $\kappa \neq \frac{2 n-1}{n}$, the change of variables

$$
e^{-2 f}=\varphi^{\frac{2(2 n-1-n \kappa)}{(2 n-1)(n-1)}}
$$

is admissible for positive $\varphi \in C^{\infty}(M)$. This change of variables involves the scaling constant and, in fact, what we have is a 1-parameter family, in the parameter $\kappa$, of these changes of variables corresponding to the family of PDEs in (3.2). Under this change, (3.2), the formulation of he sKlsc equation as a semi-linear PDE, can be transformed into a perturbation of a Schrödinger equation by a first-order non-selfadjoint term

$$
-\Delta \varphi-\frac{n \kappa}{2 n-1-n \kappa}\langle d \varphi, \theta\rangle_{\omega}+\frac{(2 n-1)(n-1)}{2(n \kappa+1-2 n)^{2}}\left(S(g)-2 \kappa \cdot S_{C}(g)\right) \varphi=0 .
$$

Since the spectrum of non-self-adjoint operators is non-real in general, in the non-zero Gauduchon degree setting we restrict our attention to conformal classes which contain a balanced representative, which we denote by $g_{b}$, so that the Lee form vanishes and the sKlsc equation based at $g_{b}$ becomes the Schrödinger equation

$$
L_{\kappa}(\varphi)=-\Delta \varphi+\frac{(2 n-1)(n-1)}{2(n \kappa+1-2 n)^{2}}\left(S\left(g_{b}\right)-2 \kappa \cdot S_{C}\left(g_{b}\right)\right) \varphi=0
$$

which has real spectrum bounded below. Since the conformal factor (3.8) cannot vanish, the sKlsc problem here becomes the question of whether there exists a scaling constant $\kappa \neq \frac{2 n-1}{n}$ for which there is a positive solution to (3.10). Since the manifold is compact, recall from Section [2.2, that this is exactly the question of whether or not there exists some scaling constant $\kappa$ for which the operator associated to the corresponding Schrödinger equation (3.10) has zero as its lowest eigenvalue. Thus, the sKlsc problem is transformed into a 1-parameter family of eigenvalue problems. 
We will denote the lowest eigenvalue of the operator $L_{\kappa}$ by $\lambda_{0}(\kappa)$. Observe that, if the conformal class contains a Kähler representative, $g_{k}$, then the sKlsc equation (3.10) based at the Kähler metric is just $-\Delta \varphi+\frac{(1-\kappa)(2 n-1)(n-1)}{(n \kappa+1-2 n)^{2}} S_{C}\left(g_{k}\right) \varphi=0$, since $S\left(g_{k}\right)=2 S_{C}\left(g_{k}\right)$. In this case, the 1-parameter family of Scrödinger equations is determined by a scaling in $\kappa$ of the fixed potential $S_{C}\left(g_{k}\right)$, and the problem can be studied in a more systematic way via the theory developed in [5]. However, if the conformal class does not contain a Kähler representative, then not only is there a scaling in $\kappa$ on the potential coming from the coefficient $\frac{(2 n-1)(n-1)}{(n \kappa+1-2 n)^{2}}$, but there is also a warping of the potential by $\kappa$ in the $S(g)-2 \kappa \cdot S_{C}(g)$ component since $S(g) \neq 2 S_{C}(g)$ for all metrics in the confomal class. While this warping leads to certain analytic complications, it also allows for proving certain existence results that are not possible to completely guarantee in the case where the conformal class contains a Kähler representative.

It is interesting to notice the similarity between the sKlsc equation (3.10), particularly in the case that the conformal class contains a Kähler representative, and the Lichnerowicz-Weitzenböck formula in spin geometry, see [16]. Also, note that the change of variables (3.8) is reminiscent of the Cole-Hopf transformation for Burgers' equation, see 9 .

The remainder of the proof separates by the Gauduchon degree of the conformal class as the behavior of the sKlsc equation, and the nature of the sKlsc question itself, differs depending upon whether it is positive or negative. The negative and positive Gauduchon degree cases are addressed below in Section 5 and Section 6 respectively.

\section{SCHRÖDINGER OPERATORS WITH SCALED AND WARPED POTENTIALS}

In Section 3, we saw that the sKlsc equation can be transformed into a 1-parameter family of Schrödinger equations whose operators are of the form

$$
L_{t}(\varphi)=-\Delta \varphi+f(t)\left(\mathcal{V}_{1}+h(t) \mathcal{V}_{2}\right) \varphi
$$

where $\mathcal{V}_{1}, \mathcal{V}_{2}: M \rightarrow \mathbb{R}$ are fixed potential functions on the $M$, and $f, h:(a, b) \rightarrow \mathbb{R}$ are functions in the parameter $t \in(a, b) \subset \mathbb{R}$ which scale and warp the fixed potentials respectively. For the sKlsc equation $\mathcal{V}_{1}=S(g), \mathcal{V}_{2}=S_{C}(g), f=\frac{(2 n-1)(n-1)}{2(n k+1-2 n)^{2}}$ and $h=2 \kappa$ as the parameter $\kappa$ ranges over the admissible range of scaling constants in each case. The parameter $t$ is used in this section, as opposed to $\kappa$, to distinguish the work here from the specific sKlsc question.

While it is the sKlsc equation which led to the consideration of such families of operators, the lack of stability that we examine here is interesting in a more general setting and is thus addressed from the perspective of a compact Riemannian manifold of arbitrary dimension. Certainly the behavior of the lowest eigenvalue of families of operators of the form (4.1) depends upon qualities of the fixed potentials $\mathcal{V}_{1}$ and $\mathcal{V}_{2}$, as well as qualities of the scaling and warping functions $f(t)$ and $h(t)$. While it is of course impossible to characterize the behavior of these eigenvalues for completely arbitrary such functions, given some reasonable assumptions of their qualities, one 
can often do this using standard analysis of the Rayleigh quotient in some cases or the work of the authors in [5], mentioned in Theorem 2.2 above.

However, there are several cases of very reasonable assumptions on the functions in the operators (4.1) for which the behavior of the lowest eigenvalue cannot be easily classified. One such case arises in our study of the sKlsc question, and is addressed in (1C) of Section 5.1 below. Many cases of the general problem actually reduce to the same thing analytically, and what this difficulty amounts to is a lack of stability in the behavior of the lowest eigenvalue. For simplicity, we state this analytic situation in terms that can be directly applied to the sKlsc problem.

Let $L_{t}(\cdot)$, where $a<t<b$, be a 1-parameter family of operators of the form (4.1) on a Riemannian manifold $(M, g)$, and denote the lowest eigenvalue by $\lambda_{0}(t)$. Assume that the scaling and warping functions

$$
\begin{aligned}
& f, h:(a, b) \subset \mathbb{R} \rightarrow \mathbb{R}^{+} \text {are positive, continuous and satisfy } \\
& \text { (i) } \lim _{t \rightarrow a} f(t)=C_{f(a)}<\infty \text { and } \lim _{t \rightarrow b} f(t)=\infty \\
& \text { (ii) } \lim _{t \rightarrow a} h(t)=C_{h(a)}<\infty \text { and } \lim _{t \rightarrow b} h(t)=C_{h(b)}<\infty
\end{aligned}
$$

(iii) $h(t)$ is strictly monotone increasing on the interval $(a, b)$,

and that the fixed potentials $\mathcal{V}_{1}, \mathcal{V}_{2} \in C^{\infty}(M)$ satisfy

$$
\begin{aligned}
& \text { (i) } \mathcal{V}_{1} \leq \mathcal{V}_{2} \\
& \text { (ii) } \int_{M} \mathcal{V}_{1}+C_{h(a)} \mathcal{V}_{2}<0
\end{aligned}
$$

From (4.3) part (ii) and the family of Rayleigh quotients associated to these operators (2.9), it is clear that for a range of $t$ close enough to $a$, the lowest eigenvalue of $L_{t}$ is strictly negative. If $\mathcal{V}_{1}+C_{h(b)} \mathcal{V}_{2}>0$ everywhere on the manifold, then from (2.9) the lowest eigenvalue for a range of $t$ close enough to $b$ is strictly positive, and thus by continuity there exists a $t^{*} \in(a, b)$ for which $\lambda_{0}\left(t^{*}\right)=0$.

Now, what we ask is the following: What if $\mathcal{V}_{1}+C_{h(b)} \mathcal{V}_{2} \geq 0$ with equality at least at one point? (It is, of course, assumed this is not identically zero.) In the case that at every point $p \in M$ where $\mathcal{V}_{1}(p)+C_{h(b)} \mathcal{V}_{2}(p)=0$, the fixed potentials both vanish themselves, i.e. $\mathcal{V}_{1}(p)=\mathcal{V}_{2}(p)=0$, then the same argument as when $\mathcal{V}_{1}+C_{h(b)} \mathcal{V}_{2}>0$ holds to show that the behavior of the lowest eigenvalue is the same as in that situation.

However, if at even one such point $p \in M$ this is not the case, in other words $\mathcal{V}_{1}(p)+C_{h(b)} \mathcal{V}_{2}(p)=0$ but neither $\mathcal{V}_{1}(p)$ nor $\mathcal{V}_{2}(p)$ vanish, then for any $\epsilon>0$

$$
\mathcal{V}_{1}(p)+h(b-\epsilon) \mathcal{V}_{2}(p)<0
$$

since $h(t)$ is strictly monotone increasing and the fixed potentials satisfy (4.3). Although this now changes sign, for small enough such $\epsilon>0$

$$
\int_{M} \mathcal{V}_{1}+h(b-\epsilon) \mathcal{V}_{2}>0
$$


and therefore, by Remark 2.4, the lowest eigenvalue $\lambda_{0}(b-\epsilon)<0$. Thus, it is not possible to guarantee the existence of a zero lowest eigenvalue using continuity and perturbative methods. These perturbative methods, though, do not allow one to understand or exercise any control over the behavior of the lowest eigenvalue for $t \in(a, b)$ away from the ends of this interval. Furthermore, there is no increasing condition on $f(t)$ and a scaling function which shrinks the entire potential away from the endpoints of $(a, b)$ is certainly admissible. Thus, perhaps there are zero and positive lowest eigenvalues there. We will show that this can never happen, and that $\lambda_{0}(t)<0$ for all $t \in(a, b)$.

Theorem 4.1. On a compact Riemannian manifold consider the 1-parameter family of Schrödinger operators

$$
L_{t}=-\Delta+f(t)\left(\mathcal{V}_{1}+h(t) \mathcal{V}_{2}\right),
$$

for $t \in(a, b)$, where the potentials satisfy (4.2) and (4.3). If $\mathcal{V}_{1}+C_{h(b)} \mathcal{V}_{2} \geq 0$ and there exists a point $p \in M$ at which

$$
\mathcal{V}_{1}(p)+C_{h(b)} \mathcal{V}_{2}(p)=0
$$

where

$$
\mathcal{V}_{1}(p) \neq 0 \text { and } \mathcal{V}_{2}(p) \neq 0
$$

then the lowest eigenvalue of $L_{t}$ is strictly negative for all $t \in(a, b)$.

Proof. The proof of this amounts to showing a lack of stability in the behavior. Recall, from the above discussion, that the lowest eigenvalues for $t$ close enough to $a$ or $b$ are negative. However, the perturbative arguments used to see this do not extend to the interior of this regime. Consider the 2-parameter family of operators

$$
L_{(t, s)}=-\Delta+f(t)\left(\mathcal{V}_{1}+h(t)\left(\mathcal{V}_{2}+s\right)\right),
$$

where $\mathcal{V}_{1}, \mathcal{V}_{2}$ and $f(t), g(t)$ satisfy the conditions of the theorem, and $s$ is a constant ranging over $-\infty<s<\infty$. Denote the lowest eigenvalue of $L_{(t, s)}$ by $\lambda_{0}(t, s)$ and the associated eigenfunction by $\varphi_{(t, s)}$. We can assume $\varphi_{(t, s)}>0$ and that $\left\|\varphi_{(t, s)}\right\|_{2}=1$ so these eigenfunctions are unique.

We begin by examining how the behavior of the lowest eigenvalue changes with $s$. Observe that, since

$$
\begin{aligned}
\lambda_{0}(t, s) \varphi_{(t, s)} & =-\Delta \varphi_{(t, s)}+f(t)\left(\mathcal{V}_{1}+h(t)\left(\mathcal{V}_{2}+s\right)\right) \varphi_{(t, s)} \\
& =\left[-\Delta \varphi_{(t, s)}+f(t)\left(\mathcal{V}_{1}+h(t) \mathcal{V}_{2}\right) \varphi_{(t, s)}\right]+h(t) f(t) s \cdot \varphi_{(t, s)} \\
& =\left(\lambda_{0}(t, 0)+h(t) f(t) s\right) \varphi_{(t, s)},
\end{aligned}
$$

for a fixed $t \in(a, b)$ the eigenfunctions corresponding to the lowest eigenvalues for all $s$ are in fact equal, i.e. for any $s_{1}, s_{2} \in(-\infty, \infty)$ the associated eigenfunctions $\varphi_{\left(t, s_{1}\right)}=\varphi_{\left(t, s_{2}\right)}$, and furthermore that the lowest eigenvalues are related by

$$
\lambda_{0}\left(t, s_{1}\right)-\lambda_{0}\left(t, s_{2}\right)=f(t) h(t)\left(s_{1}-s_{2}\right) .
$$


Therefore, since $f(t)$ and $h(t)$ are positive,

$$
\frac{\partial}{\partial s}\left(\lambda_{0}(t, s)\right)>0
$$

Now, for any $s>0$,

$$
\mathcal{V}_{1}+C_{h(b)}\left(\mathcal{V}_{2}+s\right)>0
$$

Therefore there exists a range of $\epsilon>0$ for which $\lambda_{0}(b-\epsilon, s)>0$, which in turn guarantees the existence of some $t \in(a, b)$ at which the lowest eigenvalue is zero. We will see eventually that there is a unique such $t$, but at this point there is still the possibility of several, and we denote the greatest of these by $t^{*}(s)$. In other words, for any $s>0$ the lowest eigenvalue $\lambda_{0}\left(t^{*}(s), s\right)=0$ and $t^{*}(s)$ is the greatest value in $(a, b)$ for which this is true. From (4.9) we see, while $s>0$, that

$$
\frac{d}{d s}\left(t^{*}(s)\right)<0
$$

so as $s$ increases the largest value of $t \in(a, b)$ for which the lowest eigenvalue is zero decreases. In a sense, what we find is that these $t^{*}(s)$ are foliating the regime $(a, b)$.

Finally, suppose that there exists a $t^{*}(0) \in(a, b)$ for which the lowest eigenvalue of $L_{(t, 0)}$ is zero. Notice that $L_{(t, 0)}$ is precisely the 1-parameter family of Schrödinger operators in Theorem 4.1. Then, since $(a, b)$ is follated by the $t^{*}(s)$ for $s>0$, there must be some particular $s>0$ for which $\lambda_{0}\left(t^{*}(0), 0\right)=\lambda_{0}\left(t^{*}(s), s\right)=0$ and $t^{*}(0)=t^{*}(s)$. However, from (4.8), we see that this cannot occur.

\section{Negative Gauduchon Degree CASE}

Recall, from (3.4), that the admissible regime of scaling constants when the conformal class has negative Gauduchon degree is $\kappa \in(1, \infty)$. The degenerate scaling constant $\kappa=\frac{2 n-1}{n}$ separates this into two disjoint intervals $\left(1, \frac{2 n-1}{n}\right) \sqcup\left(\frac{2 n-1}{n}, \infty\right)$ and, in turn, the sKlsc question here separates into two problems by restricting the sKlsc equation (3.10) to each of these intervals. The study of these questions further separates depending upon whether the conformal class does or does not contain a Kähler representative. Interestingly, the difference primarily lies in the range of scaling constants $\kappa \in\left(1, \frac{2 n-1}{n}\right)$. The sKlsc question is answered in the non-degenerate setting in Section 5.1 and Section [5.2, and is addressed for the degenerate scaling constant in Section 5.3.

5.1. When $[g]$ does not contain a Kähler representative. Consider, first, the sKlsc question in the case that there exists a balanced representative, $g_{b}$, of the conformal class, but no Kähler representative. Base the family of sKlsc equations at this balanced metric, so the non-self-adjoint term vanish, and they take the form of (3.10). The result makes use of the continuity of the lowest eigenvalue of the family of operators $L_{\kappa}$ and relies upon a detailed analysis of the behavior of these eigenvalues throughout the admissible regime of scaling constants $\left(1, \frac{2 n-1}{n}\right) \sqcup\left(\frac{2 n-1}{n}, \infty\right)$, most importantly, around the ends of these open intervals. The behavior is straightforward 
to understand for $\kappa$ "close enough" to 1 and $\infty$, and is discussed forthwith. However, the analysis is quite subtle for $\kappa$ in a neighborhood of the degenerate scaling constant.

For $\kappa$ near 1 , since $\int_{M} S\left(g_{b}\right)<2 \int_{M} S_{C}\left(g_{b}\right)<0$, there exists an $\epsilon^{*}>0$ small enough so that, for all $\epsilon \in\left(0, \epsilon^{*}\right)$, the perturbation

$$
\int_{M} S\left(g_{b}\right)-2(1+\epsilon) S_{C}\left(g_{b}\right)<0
$$

Therefore, the lowest eigenvalue of the operators $L_{1+\epsilon}$ satisfy $\lambda_{0}(1+\epsilon)<0$. Similarly, any $\kappa>>\frac{2 n-1}{n}$ large enough ensures that $\int_{M} S\left(g_{b}\right)-2 \kappa \cdot S_{C}\left(g_{b}\right)>0$. Then, since $\int_{M} S\left(g_{b}\right)-2 \kappa \cdot S_{C}\left(g_{b}\right)$ is an increasing function of $\kappa$ and

$$
\lim _{\kappa \mapsto \infty} \frac{(2 n-1)(n-1)}{2(n \kappa+1-2 n)^{2}}\left(S\left(g_{b}\right)-2 \kappa \cdot S_{C}\left(g_{b}\right)\right)=0,
$$

there must exist some range of scaling constants $\left(\kappa^{*}, \infty\right) \subset\left(\frac{2 n-1}{n}, \infty\right)$ for which the operators $L_{\kappa}$ have lowest eigenvalues $\lambda_{0}(\kappa)>0$ for all $\kappa \in\left(\kappa^{*}, \infty\right)$. This follows from Theorem 2.2 and Remark 2.4 if $S_{C}\left(g_{b}\right)$ changes sign on the manifold, and directly from examining the Rayleigh quotient if $S_{C}\left(g_{b}\right)$ is non-positive.

The behavior of the lowest eigenvalues for the operators $L_{\kappa}$ around the degenerate scaling constant $\kappa=\frac{2 n-1}{n}$ depends upon certain qualities of $S\left(g_{b}\right)-2\left(\frac{2 n-1}{n}\right) S_{C}\left(g_{b}\right)$ as a function on the manifold. The question first separates into cases depending upon whether $\int_{M} S\left(g_{b}\right)-2\left(\frac{2 n-1}{n}\right) S_{C}\left(g_{b}\right)$ is positive, zero or negative.

(1) When $\int_{M} S\left(g_{b}\right)-2\left(\frac{2 n-1}{n}\right) S_{C}\left(g_{b}\right)>0$, small perturbations of the scaling constant preserve positivity, i.e. for $\epsilon>0$ small enough

$$
\int_{M} S\left(g_{b}\right)-2\left(\frac{2 n-1}{n} \pm \epsilon\right) S_{C}\left(g_{b}\right)>0 .
$$

The behavior of the lowest eigenvalue separates this case into three subcases depending upon if $S-2\left(\frac{2 n-1}{n}\right) S_{C}$ changes sign, is strictly positive or is nonnegative and equals zero at least once on the manifold.

(a) If $S\left(g_{b}\right)-2\left(\frac{2 n-1}{n}\right) S_{C}\left(g_{b}\right)$ changes sign on the manifold, then for a range of $\epsilon>0$ small enough, $S\left(g_{b}\right)-2\left(\frac{2 n-1}{n} \pm \epsilon\right) S_{C}\left(g_{b}\right)$ changes sign on the manifold as well and, from Remark [2.4, we see that $\lambda_{0}\left(\frac{2 n-1}{n} \pm \epsilon\right)<0$. Thus, recalling $\lambda_{0}\left(\kappa>>\frac{2 n-1}{n}\right)>0$ for $\kappa$ large enough, there is a scaling constant $\kappa \in\left(\frac{2 n-1}{n}, \infty\right)$ for which a solution to the sKlsc problem exists.

Remark 5.1. In the regime $\left(1, \frac{2 n-1}{n}\right)$ a solution is not guaranteed to exist since the lowest eigenvalues for scaling constants close to 1 and $\frac{2 n-1}{n}$ are negative. However, it is certainly possible for there to be a solution in this regime as, for $\epsilon_{1}>0$ and $\epsilon_{2}>0$ small enough, $\lambda_{0}\left(1+\epsilon_{1}\right)<0$ due to the fact that $\int_{M} S\left(g_{b}\right)-2\left(1+\epsilon_{1}\right) S_{C}\left(g_{b}\right)<0$ for , while $\lambda_{0}\left(\frac{2 n-1}{n}-\epsilon_{2}\right)<0$ follows from Remark 2.4 because $S\left(g_{b}\right)-2\left(\frac{2 n-1}{n}-\epsilon_{2}\right) S_{C}\left(g_{b}\right)$ changes sign while $\int_{M} S\left(g_{b}\right)-2\left(1+\epsilon_{2}\right) S_{C}\left(g_{b}\right)>0$, and $\lim _{\epsilon_{2} \mapsto 0} \frac{1}{\left(n\left(\frac{2 n-1}{n}-\epsilon_{2}\right)+1-2 n\right)^{2}}=\infty$. 
(b) If $S\left(g_{b}\right)-2\left(\frac{2 n-1}{n}\right) S_{C}\left(g_{b}\right)>0$, then for a range of $\epsilon>0$ small enough,

$$
S\left(g_{b}\right)-2\left(\frac{2 n-1}{n} \pm \epsilon\right) S_{C}\left(g_{b}\right)>0
$$

as well, so $\lambda_{0}\left(\frac{2 n-1}{n} \pm \epsilon\right)>0$. Thus, recalling that $\lambda_{0}(1+\epsilon)<0$ for $\epsilon>0$ small enough, there is a scaling constant $\kappa \in\left(1, \frac{2 n-1}{n}\right)$ for which a solution to the sKlsc problem exists. However, there are no solution to the sKlsc problem for scaling constants in the regime $\left(\frac{2 n-1}{n}, \infty\right)$ since $S\left(g_{b}\right)-2 \kappa \cdot S_{C}\left(g_{b}\right)$ is an increasing function, and therefore positive, on this entire range.

(c) Suppose $S\left(g_{b}\right)-2\left(\frac{2 n-1}{n}\right) S_{C}\left(g_{b}\right) \geq 0$, with $\left.S\left(g_{b}\right)\right|_{p}-\left.2\left(\frac{2 n-1}{n}\right) S_{C}\left(g_{b}\right)\right|_{p}=0$ at least at one point $p \in M$. If at each such point $p \in M$, this is zero because $\left.S\left(g_{b}\right)\right|_{p}=\left.S_{C}\left(g_{b}\right)\right|_{p}=0$, the same argument as in (1b) above shows that there is a scaling constant $\kappa \in\left(1, \frac{2 n-1}{n}\right)$ for which a solution to the sKlsc problem exists, and that there are no solution to the sKlsc problem for scaling constants in the regime $\left(\frac{2 n-1}{n}, \infty\right)$. However, if at any point $p \in M$ at which this vanishes neither of the scalar curvatures vanish, in other words

$$
\left.S\left(g_{b}\right)\right|_{p}-\left.2\left(\frac{2 n-1}{n}\right) S_{C}\left(g_{b}\right)\right|_{p}=0
$$

but

$$
\left.S\left(g_{b}\right)\right|_{p} \neq 0 \text { and }\left.S_{C}\left(g_{b}\right)\right|_{p} \neq 0
$$

then there are no solutions to the sKlsc problem for scaling constants in the union of the regimes $\left(1, \frac{2 n-1}{n}\right) \sqcup\left(\frac{2 n-1}{n}, \infty\right)$. This is seen for the regime $\left(\frac{2 n-1}{n}, \infty\right)$ since $S\left(g_{b}\right)-2 \kappa \cdot S_{C}\left(g_{b}\right)$ is an increasing function of $\kappa$, and therefore non-negative on this entire range, so $\lambda_{0}(\kappa)>0$ here. For the regime of scaling constants $\left(1, \frac{2 n-1}{n}\right)$ the non-existence follows immediately from Theorem 4.1, which concerns the lack of stability for Schrödinger operators with such a warping and scaling of the potential.

(2) When $\int_{M} S\left(g_{b}\right)-2\left(\frac{2 n-1}{n}\right) S_{C}\left(g_{b}\right)=0$, then

$$
\int_{M} S\left(g_{b}\right)-2 \kappa \cdot S_{C}\left(g_{b}\right)\left\{\begin{array}{ll}
<0 & \text { for } \kappa<\frac{2 n-1}{n} \\
>0 & \text { for } \kappa>\frac{2 n-1}{n}
\end{array} .\right.
$$

Thus, there are no solutions to the sKlsc problem for $\kappa \in\left(1, \frac{2 n-1}{n}\right)$ as $\lambda_{0}(\kappa)<0$ over this entire regime. The question of existence for $\kappa \in\left(\frac{2 n-1}{n}, \infty\right)$ depends upon whether or not $S\left(g_{b}\right)-2\left(\frac{2 n-1}{n}\right) S_{C}\left(g_{b}\right)$ changes sign.

(a) If $S\left(g_{b}\right)-2\left(\frac{2 n-1}{n}\right) S_{C}\left(g_{b}\right)$ changes sign, by the same argument as in (1a), there is a scaling constant $\kappa \in\left(\frac{2 n-1}{n}, \infty\right)$ for which a solution to the sKlsc 
problem exists.

(b) If $S\left(g_{b}\right)-2\left(\frac{2 n-1}{n}\right) S_{C}\left(g_{b}\right)$ does not change sign, then it is necessarily identically zero since it integrates to zero. Therefore, the balanced metric is an sKlsc metric with $\kappa=\frac{2 n-1}{n}$. Furthermore, this is the unique sKlsc metric, up to scale, in the conformal class.

(3) When $\int_{M} S\left(g_{b}\right)-2\left(\frac{2 n-1}{n}\right) S_{C}\left(g_{b}\right)<0$, for a range of $\epsilon>0$ small enough

$$
\int_{M} S\left(g_{b}\right)-2\left(\frac{2 n-1}{n}+\epsilon\right) S_{C}\left(g_{b}\right)<0
$$

as well, so $\lambda_{0}\left(\frac{2 n-1}{n}+\epsilon\right)<0$. Thus, recalling that $\lambda_{0}\left(\kappa>>\frac{2 n-1}{n}\right)>0$, there is a scaling constant $\kappa \in\left(\frac{2 n-1}{n}, \infty\right)$ for which a solution to the sKlsc problem exists. However, there is no solution for any $\kappa \in\left(1, \frac{2 n-1}{n}\right)$ as $\int_{M} S\left(g_{b}\right)-2 \kappa \cdot S_{C}\left(g_{b}\right)<0$, so $\lambda_{0}(\kappa)<0$ for all scaling constants in this regime.

5.2. When $[g]$ contains a Kähler representative. In the case that there does exist a Kähler representative, $g_{k}$, of the conformal class, recall that the family of sKlsc equations based at this metric simplifies to $-\Delta \varphi+\frac{(1-\kappa)(2 n-1)(n-1)}{(n \kappa+1-2 n)^{2}} S_{C}\left(g_{k}\right) \cdot \varphi=0$, since for a Kähler metric $S\left(g_{k}\right)=2 S_{C}\left(g_{k}\right)$. This can be viewed as a 1-parameter family of Schrödinger equations with a scaling of a fixed potential, $S_{C}\left(g_{k}\right)$, in the parameter $\kappa$. In this situation, the sKlsc problem is exactly the same question for both of the regimes $\kappa_{1} \in\left(1, \frac{2 n-1}{n}\right)$ and $\kappa_{2} \in\left(\frac{2 n-1}{n}, \infty\right)$ as there is a bijective correspondence between given by the operators restricted to these given by setting

$$
\frac{\left(\kappa_{1}-1\right)(2 n-1)(n-1)}{\left(n \kappa_{1}+1-2 n\right)^{2}}=\frac{\left(\kappa_{2}-1\right)(2 n-1)(n-1)}{\left(n \kappa_{2}+1-2 n\right)^{2}},
$$

and solving to extract the relationship

$$
\left(\kappa_{1}-1\right)\left(\kappa_{2}-1\right)=\frac{(n-1)^{2}}{n^{2}}
$$

between $\kappa_{1}$ and $\kappa_{2}$ in their respective domains. Then, solving for either, observe that the derivative with respect to the other is nonvanishing in the appropriate regime. Therefore, a solution to the problem in one regime guarantees a solution in the other since the operators $L_{\kappa_{1}}=L_{\kappa_{2}}$ for each unique pair of scaling constants $\kappa_{1} \in\left(1, \frac{2 n-1}{n}\right)$ and $\kappa_{2} \in\left(\frac{2 n-1}{n}, \infty\right)$ that satisfy (5.3). It is easy to check that $L_{\kappa_{2}}=-\Delta+\frac{\left(1-\kappa_{2}\right)(2 n-1)(n-1)}{\left(n \kappa_{2}+1-2 n\right)^{2}} S_{C}\left(g_{k}\right)$ satisfies the conditions of Theorem 2.2 , and thus the existence and uniqueness results of Theorem 1.5 part (1) follow accordingly. The relationship between the conformal factors follows from (5.3) and (3.8).

Remark 5.2. Although it is the same positive function $\varphi$ that provides the solution to the sKlsc equation in both the regimes $\left(1, \frac{2 n-1}{n}\right)$ and $\left(\frac{2 n-1}{n}, \infty\right)$, and this function is unique up to scale, it is important to note that the actual conformal factors (3.8) used to obtain the sKlsc metric for each of the scaling constants, $\kappa_{1}$ and $\kappa_{2}$, are different 
powers of $\varphi$, and thus the behavior of the resulting scalar curvatures, while both being sKlsc with their respective scaling constants, can differ greatly.

5.3. The degenerate scaling constant. Recall that, when $\kappa=\frac{2 n-1}{n}$, the sKlsc equation (3.2) degenerates into the first order non-linear autonomous Hamilton-Jacobi equation

$$
|\nabla f|^{2}-\frac{1}{n-1}\langle d f, \theta\rangle_{\omega}=\frac{S(g)-2\left(\frac{2 n-1}{n}\right) S_{C}(g)}{2(2 n-1)(n-1)} .
$$

A solution sought for here is not required to be positive as this formulation of the sKlsc equation is with respect to the conformal factor $e^{-2 f}$. There are a variety of likely obstructions to the existence of sKlsc metrics with this scaling constant.

By basing (5.4) at the Gauduchon metric, $g_{G}$, an integrating, we find that there is an immediate obstruction to the existence of non-Kähler sKlsc metrics unless the Gauduchon degree is negative since $\int_{M}|\nabla f|^{2}-\frac{1}{n-1}\langle d f, \theta\rangle_{\omega}=\int_{M}|\nabla f|^{2} \geq 0$, because the Lee form is co-closed, and

$$
\int_{M} S\left(g_{G}\right)-2\left(\frac{2 n-1}{n}\right) S_{C}\left(g_{G}\right)>0 \text { only if } \int_{M} S_{C}\left(g_{G}\right)<0 .
$$

If the conformal class contains a balanced representative, (5.4) becomes

$$
|\nabla f|^{2}=\frac{S\left(g_{b}\right)-2\left(\frac{2 n-1}{n}\right) S_{C}\left(g_{b}\right)}{2(2 n-1)(n-1)}=\frac{\left(\frac{1-n}{n}\right) S_{C}\left(g_{b}\right)-\frac{1}{4}\left|T\left(g_{b}\right)\right|^{2}}{(2 n-1)(n-1)},
$$

see [19, Theorem 1.6], and observe that there is an obstruction to the existence of non-Kähler sKlsc metrics unless $S_{C}\left(g_{b}\right)$ is non-positive and not identically zero. Also, notice that for there to possible exist a solution, $S\left(g_{b}\right)-2\left(\frac{2 n-1}{n}\right) S_{C}\left(g_{b}\right)$ must vanish at least twice on the manifold.

If the conformal class contains a Kähler representative, $g_{k}$, the degenerate sKlsc equation (5.6) becomes

$$
|\nabla f|^{2}=-\frac{S_{C}\left(g_{k}\right)}{n(2 n-1)}
$$

then there is the further obstruction that $S_{C}\left(g_{k}\right)$ must equal zero at least at two points on the manifold to the existence of sKlsc metrics. This is due to the fact that, if there exists a solution $f$, it cannot be constant and must attain distinct maximums and minimums on the manifold. On the other hand, recall from Section 5, if $S_{C}\left(g_{k}\right)$ is non-positive and not identically zero and the conformal class contains a Kähler representative, then this degenerate scaling constant $\kappa=\frac{2 n-1}{n}$ is the only scaling constant for which there could possibly exist non-Kähler sKlsc metrics.

Remark 5.3. Although there are many compact balanced manifolds with non-positive Chern scalar curvature, and with $S\left(g_{b}\right)-2\left(\frac{2 n-1}{n}\right) S_{C}\left(g_{b}\right)$ vanishing at least twice on the manifold, it is rare for classical solutions to equations such as (5.6) to exist, see [10. Interestingly, due to the sparseness of solutions for particular values of $\kappa$ limiting to $\frac{2 n-1}{n}$, it is even rare for viscosity solutions (with lower regularity) to exist as in [7]. 


\section{Positive Gauduchon Degree Case}

Recall, from (3.4), that the admissible regime of scaling constants when the conformal class has positive Gauduchon degree is $\kappa \in(-\infty, 1)$. For similar reasons as in the negative Gauduchon degree case, the study of the sKlsc question here separates depending upon whether the conformal class does or does not contain a Kähler representative. It is interesting to note, that there is a more stark contrast here between existence results each of these cases than in the negative Gauduchon degree setting.

6.1. When $[g]$ does not contain a Kähler representative. Consider, first, the sKlsc question in the case that there exists a balanced representative, $g_{b}$, of the conformal class, but no Kähler representative. Base the family of sKlsc equations at this balanced metric, so the non-self-adjoint term vanish, and they take the form of (3.10). Since

$$
\int_{M} S\left(g_{b}\right)-2 S_{C}\left(g_{b}\right)<0 \text { and } \int_{M} S_{C}\left(g_{b}\right)>0
$$

there exists an $\epsilon^{*}>0$ small enough so that, for all $\epsilon \in\left(0, \epsilon^{*}\right)$, the perturbation

$$
\int_{M} S\left(g_{b}\right)-2(1-\epsilon) S_{C}\left(g_{b}\right)<0 .
$$

Therefore, the lowest eigenvalue of the operators $L_{1-\epsilon}$ satisfy $\lambda_{0}(1-\epsilon)<0$. Next, observe that any $\kappa<<0$ negative enough ensures that $\int_{M} S\left(g_{b}\right)-2 \kappa \cdot S_{C}\left(g_{b}\right)>0$, and also that

$$
\lim _{\kappa \mapsto-\infty} \frac{(2 n-1)(n-1)}{2(n \kappa+1-2 n)^{2}}\left(S\left(g_{b}\right)-2 \kappa \cdot S_{C}\left(g_{b}\right)\right)=0 .
$$

Therefore, there must exist some range of scaling constants $\left(-\infty, \kappa^{*}\right) \subset(-\infty, 1)$ for which the operators $L_{\kappa}$ have lowest eigenvalues $\lambda_{0}(\kappa)>0$ for all $\kappa \in\left(-\infty, \kappa^{*}\right)$. This follows from Theorem 2.2 if $S_{C}\left(g_{b}\right)$ changes sign on the manifold, and directly from examining the Rayleigh quotient if $S_{C}\left(g_{b}\right)$ is non-negative. Thus a solution to the sKlsc problem with scaling constant in the regime $\kappa \in\left(\infty, \frac{2 n-1}{n}\right)$ is guaranteed.

6.2. When $[g]$ contains a Kähler representative. In the case that there does exist a Kähler representative, $g_{k}$, of the conformal class, recall that the family of sKlsc equations based at this metric simplifies to $-\Delta \varphi+\frac{(1-\kappa)(2 n-1)(n-1)}{(n \kappa+1-2 n)^{2}} S_{C}\left(g_{k}\right) \cdot \varphi=0$, since for a Kähler metric $S\left(g_{k}\right)=2 S_{C}\left(g_{k}\right)$. By integrating this equation, observe that no non-Kähler solution to the sKlsc problem exists if $S_{C}\left(g_{k}\right) \geq 0$ since $\varphi>0$. Hence, we now assume that $S_{C}\left(g_{k}\right)$ changes sign on the manifold. However, it is not analytically possible to exercise the same control over the behavior of the lowest eigenvalue for scaling constants close to 1 , in other words for $\kappa \in(1-\epsilon, 1)$ for small $\epsilon>0$, as in situation where the conformal class does not contain a Kähler metric. This is because here the the potential function will always have positive average. Examining 
the multiplier of $S_{C}\left(g_{k}\right)$ in the potential function, we find that

$$
\max _{\kappa<1} \frac{(1-\kappa)(2 n-1)(n-1)}{(n \kappa+1-2 n)^{2}}=\frac{2 n-1}{4 n},
$$

where this maximum is achieved at $\kappa=\frac{1}{n}$. Therefore, even if $S_{C}\left(g_{k}\right)$ changes sign, the sKlsc problem here cannot be formulated in a way, analogous to the proof of the negative Gauduchon degree case above as to satisfy the conditions of Theorem 2.2 since the multiplier on the potential function, which is a function of $\kappa<1$, is bounded.

More specifically, while Theorem 2.1 holds to guarantee the existence of open intervals of scaling constants, $\kappa<1$, near both 1 and $-\infty$ for which the lowest eigenvalue of

$$
-\Delta+\frac{(1-\kappa)(2 n-1)(n-1)}{(n \kappa+1-2 n)^{2}} S_{C}\left(g_{k}\right)
$$

is strictly positive, since (6.4) is bounded it is not possible to ensure the existence of a member of this family with negative lowest eigenvalue which is necessary in the proof of Theorem 2.2 as it relies on continuity of the lowest eigenvalue in the parameter $\kappa$. However, Theorem 2.1 can be used as an obstruction to the occurrence of a negative lowest eigenvalue, for if $\frac{2 n-1}{4 n}$, which recall is the maximum of the multiplier on the potential, satisfies the inequality

$$
\frac{2 n-1}{4 n} \leq \frac{\int_{M} S_{C}\left(g_{k}\right)}{P\left\|S_{C}\left(g_{k}\right)\right\|_{\infty}\left(4 \operatorname{Vol}(M)\left\|S_{C}\left(g_{k}\right)\right\|_{\infty}+\int_{M} S_{C}\left(g_{k}\right)\right)},
$$

then the lowest eigenvalue of this family of Schrödinger operators is always positive.

Note though, this does not preclude the existence of a scaling constant $\kappa<1$ for which the lowest eigenvalue is negative or zero. In fact, in certain instances we know that non-Kähler solutions to sKlsc problem here do exist. This is seen when $\kappa=\frac{2 n-1}{n^{2}}$, as the sKlsc equation (3.10) becomes

$$
-\Delta \varphi+\left(\frac{n-1}{2 n-1}\right) S_{C}\left(g_{k}\right) \cdot \varphi=0
$$

and the existence of a positive solution to this is equivalent to the existence of a Riemannian scalar-flat metric in the conformal class of $g_{k}$ (recall that $S\left(g_{k}\right)=2 S_{C}\left(g_{k}\right)$ and the real dimension is $2 n$ ). Note, though, if there does exist a positive solution $\varphi$ to (6.7), since the conformal factor to obtain the sKlsc metric would be $\varphi^{\frac{2}{n}}$, recall (3.8), the resulting sKlsc metric would not be Riemannian-scalar-flat as the conformal factor to obtain that metric would be $\varphi^{\frac{2}{n-1}}$.

Remark 6.1. Interestingly, from this along with (3.3), it is easy to see that if there is a Kähler manifold, of complex dimension $n \geq 2$, that is conformal to a nonKähler Riemannian-scalar-flat metric, then the conformal class necessarily has positive Gauduchon degree.

While there do exist solutions to the sKlsc problem in certain instances when the scalar curvature of the initial Kähler metric changes sign and satisfies $\int_{M} S_{C}\left(g_{k}\right)>0$, it is not at all clear whether solutions should exist for every such manifold, much less 
the number of solutions that may exist. In fact, the authors believe that there do exist compact Kähler manifolds, satisfying these scalar curvature conditions, which admit no conformal non-Kähler sKlsc metrics. This is loosely reminiscent of the positive Gauduchon degree case for the Chern Yamabe problem [1].

\section{REFERENCES}

[1] Daniele Angella, Simone Calamai, and Cristiano Spotti. On Chern-Yamabe Problem. to appear in Math. Res. Lett., 2015.

[2] Melvyn S. Berger. On Hermitian structures of prescribed nonpositive Hermitian scalar curvature. Bull. Amer. Math. Soc., 78:734-736, 1972.

[3] Arthur L. Besse. Einstein manifolds. Springer-Verlag, Berlin, 1987.

[4] Michael G. Dabkowski and Michael T. Lock. An equivalence of scalar curvatures on Hermitian manifolds. to appear in J. Geom. Anal., 2015.

[5] Michael G. Dabkowski and Michael T. Lock. The lowest eigenvalue of Schrödinger operators on compact manifolds. arXiv.org:1508.02755, 2015.

[6] Heberto del Rio and Santiago R. Simanca. The Yamabe problem for almost Hermitian manifolds. J. Geom. Anal., 13(1):185-203, 2003.

[7] Philippe Delanoë. Viscosity solutions of eikonal and Lie equations on compact manifolds. Ann. Global Anal. Geom., 7(2):79-83, 1989.

[8] Jean Dolbeault, Maria J. Esteban, Ari Laptev, and Michael Loss. Spectral properties of Schrödinger operators on compact manifolds: rigidity, flows, interpolation and spectral estimates. C. R. Math. Acad. Sci. Paris, 351(11-12):437-440, 2013.

[9] Lawrence C. Evans. Partial differential equations, volume 19 of Graduate Studies in Mathematics. American Mathematical Society, Providence, RI, second edition, 2010.

[10] Albert Fathi. Weak KAM from a PDE point of view: viscosity solutions of the Hamilton-Jacobi equation and Aubry set. Proc. Roy. Soc. Edinburgh Sect. A, 142(6):1193-1236, 2012.

[11] Paul Gauduchon. Le théorème de l'excentricité nulle. C. R. Acad. Sci. Paris Sér. A-B, 285(5):A387-A390, 1977.

[12] Paul Gauduchon. La 1-forme de torsion d'une variété hermitienne compacte. Math. Ann., 267(4):495-518, 1984.

[13] Alexander Grigor'yan, Nikolai Nadirashvili, and Yannick Sire. A lower bound for the number of negative eigenvalues of Schrödinger operators. J. Differential Geom., 102(3):395-408, 2016.

[14] Alexander Grigor'yan, Yuri Netrusov, and Shing-Tung Yau. Eigenvalues of elliptic operators and geometric applications. In Surveys in differential geometry. Vol. IX, Surv. Differ. Geom., IX, pages 147-217. Int. Press, Somerville, MA, 2004.

[15] Daniel Huybrechts. Complex geometry. Universitext. Springer-Verlag, Berlin, 2005. An introduction.

[16] André Lichnerowicz. Spineurs harmoniques. C. R. Acad. Sci. Paris, 257:7-9, 1963.

[17] Ke-Feng Liu and Xiao-Kui Yang. Geometry of Hermitian manifolds. Internat. J. Math., 23(6):1250055, 40, 2012.

[18] Ke-Feng Liu and Xiao-Kui Yang. Ricci curvatures on Hermitian manifolds. arXiv.org:1404.2481, 2014.

[19] M. L. Michelsohn. On the existence of special metrics in complex geometry. Acta Math., 149(34):261-295, 1982.

[20] Erwin Schrödinger. An undulatory theory of the mechanics of atoms and molecules. Phys. Rev., 28(6):1049,1070, 1926.

[21] Valentino Tosatti. Non-Kähler Calabi-Yau manifolds. In Analysis, complex geometry, and mathematical physics: in honor of Duong H. Phong, volume 644 of Contemp. Math., pages 261-277. Amer. Math. Soc., Providence, RI, 2015. 
Dept. of Mathematics, Lawrence Technological University, Southfield, Mi, 48075

E-mail address: mdabkowsk@ltu.edu

Dept. of Mathematics, University of Texas, Austin, TX, 78712

E-mail address: mlock@math.utexas.edu 